\title{
Set-Valued Functions of Bounded Generalized Variation and Set-Valued Young Integrals
}

\author{
Mariusz Michta $^{1} \cdot$ Jerzy Motyl $^{1}$ \\ Received: 8 June 2020 / Revised: 21 October 2020 / Accepted: 11 November 2020 / \\ Published online: 8 December 2020 \\ (c) The Author(s) 2020
}

\begin{abstract}
The paper deals with some properties of set-valued functions having bounded Riesz $p$-variation. Set-valued integrals of Young type for such multifunctions are introduced. Selection results and properties of such set-valued integrals are discussed. These integrals contain as a particular case set-valued stochastic integrals with respect to a fractional Brownian motion, and therefore, their properties are crucial for the investigation of solutions to stochastic differential inclusions driven by a fractional Brownian motion.
\end{abstract}

Keywords Hölder continuity - Set-valued function - Set-valued Riesz p-variation · Set-valued Young integral · Selection · Generalized Steiner center

Mathematics Subject Classification (2020) Primary 26A33; Secondary 26A16 . $26 \mathrm{~A} 45 \cdot 28 \mathrm{~B} 20 \cdot 47 \mathrm{H} 04$

\section{Introduction}

Since the pioneering work of Aumann in 1965 [6], the notion of set-valued integrals for multivalued functions has attracted the interest of many authors from both theoretical and practical points of view. In particular, the theory has been developed extensively, among others, with applications to optimal control theory, mathematical economics, theory of differential inclusions and set-valued differential equations, see, e.g., $[1,3,4,21,23,29]$. Later, the notion of the integral for set-valued functions has been extended to a stochastic case, where set-valued Itô integrals have been studied.

$凶$ Jerzy Motyl

j.motyl@wmie.uz.zgora.pl

Mariusz Michta

m.michta@wmie.uz.zgora.pl

1 Faculty of Mathematics, Computer Science and Econometrics, University of Zielona Góra, Szafrana 4a, 65-516 Zielona Gora, Poland 
Moreover, concepts of set-valued integrals, both deterministic and stochastic, were used to define the notion of fuzzy integrals applied in the theory of fuzzy differential equations, e.g., [14,24]. On the other hand, in a single-valued case, one can consider integration with respect to integrators such as fractional Brownian motion which has Hölder continuous sample paths. In some cases, such integrals can be understood in the sense of Young [30]. Controlled differential equations driven by Young integrals have been studied by Lejay in [25]. A more advanced approach to controlled differential equations is based on the rough path integration theory initiated by T. Lions [26] and further examined in $[12,17]$. Control and optimal control problems inspired the intensive expansion of differential and stochastic set-valued inclusions theory. Thus, it seems reasonable to investigate also differential inclusions driven by a fractional Brownian motion and Young-type integrals also. Recently, in [7] the authors considered a Young-type differential inclusion, where solutions were understood as Young integrals of appropriately regular selections of multivalued right-hand side. Set-valued Aumann or Itô-type integrals are useful tools in the investigation of properties of solution sets to differential or stochastic inclusions and set-valued equations [2,15,16,22]. Therefore, it is quite natural to introduce set-valued Young-type integrals. Motivated by this, the aim of this work is to introduce such set-valued integrals and to investigate their properties, especially these which seem to be useful in the Young set-valued inclusions theory. It is known that three of properties of Aumann set-valued integrals are crucial in the differential inclusions theory. Namely, they are the existence of a Castaing representation of the set of integrable selectors, decomposability of this set and valuation of a Hausdorff distance between set-valued integrals by the distance between integrated multifunctions (see, e.g., [20]).

Set-valued Young integrals considered in the paper deal with the class of set-valued functions having a bounded Riesz $p$-variation. Such integrals contain as a particular case set-valued stochastic integrals with respect to a fractional Brownian motion. Therefore, in our opinion, their properties are crucial not only for the existence of solutions to stochastic differential inclusions and set-valued stochastic differential equations driven by a fractional Brownian motion but also for useful properties of their solution sets.

The paper is organized as follows. In Sect. 2, we define a space of set-valued functions of a finite Riesz $p$-variation. Section 3 deals with the properties of sets of appropriately regular selections of such set-valued functions. Here, we shall establish a new type of decomposability for sets of functions with a finite Riesz $p$-variation as well as their integral property. Finally, in Sect. 4, we introduce a set-valued Youngtype integral which is based on the sets of selections examined in Sect. 3. We shall investigate properties of this set-valued integral.

\section{Finite $p$-Variation Set-Valued Functions}

Let $(X,\|\cdot\|)$ be a Banach space. Denote by $\operatorname{Comp}(X)$ and $\operatorname{Conv}(X)$ the families of all nonempty and compact, and nonempty compact and convex subsets of $X$, respectively. 
The Hausdorff metric $H_{X}$ in $\operatorname{Comp}(X)$ is defined by

$$
H_{X}(B, C)=\max \left\{\bar{H}_{X}(B, C), \bar{H}_{X}(C, B)\right\},
$$

where $\bar{H}_{X}(B, C)=\sup _{b \in B} \operatorname{dist}_{X}(b, C)=\sup _{b \in B} \inf _{c \in C}\|c-b\|_{X}$. If $X$ is separable, then the space $\left(\operatorname{Comp}(X), H_{X}\right)$ is a Polish space and $\left(\operatorname{Conv}(X), H_{X}\right)$ is its closed subspace. For $B, C, D, E \in \operatorname{Comp}(X)$, we have

$$
H_{X}(B+C, D+E) \leq H_{X}(B, D)+H_{X}(C, E)
$$

where $B+C:=\{b+c: b \in B, c \in C\}$ denotes the Minkowski sum of $B$ and $C$. Moreover, for $B, C, D \in \operatorname{Conv}(X)$, the equality

$$
H_{X}(B+D, C+D)=H_{X}(B, C)
$$

holds, see, e.g., [23] for details.

We use the notation

$$
\|A\|_{X}:=H_{X}(A,\{0\})=\sup _{a \in A}\|a\|_{X} \text { for } A \in \operatorname{Conv}(X) .
$$

Let $T>0$ and $\beta \in(0,1]$. For every function $f:[0, T] \rightarrow X$, we define

$$
\|f\|_{\infty}=\sup _{t \in[0, T]}\|f(t)\|_{X} \text { and } M_{\beta}(f)=\sup _{0 \leq s<t \leq T} \frac{\|f(t)-f(s)\|_{X}}{(t-s)^{\beta}} .
$$

By $\mathcal{C}^{\beta}(X)$, we denote the space of $\beta$-Hölder-continuous ( or shortly $\beta$-Hölder) functions with a finite norm

$$
\|f\|_{\beta}:=\|f\|_{\infty}+M_{\beta}(f) .
$$

It can be shown that $\left(\mathcal{C}^{\beta},\|\cdot\|_{\beta}\right)$ is a Banach space. Similarly, for a set-valued function $F:[0, T] \rightarrow \operatorname{Comp}(X)$, let

$$
\|F\|_{\beta}:=\|F\|_{\infty}+M_{\beta}(F)
$$

where

$$
\|F\|_{\infty}=\sup _{t \in[0, T]}\|F(t)\|_{X} \text { and } M_{\beta}(F)=\sup _{0 \leq s<t \leq T} \frac{H_{X}(F(t), F(s))}{(t-s)^{\beta}} .
$$

A set-valued function $F$ is said to be $\beta$-Hölder if $\|F\|_{\beta}<\infty$. By $\mathcal{C}^{\beta}(\operatorname{Comp}(X))$, we denote the space of all such set-valued functions. The space of $\beta$-Hölder set-valued functions having compact and convex values will be denoted by $\mathcal{C}^{\beta}(\operatorname{Conv}(X))$.

Let $(E, d)$ be a metric space. For every $0 \leq a<b \leq T$, by $\Pi_{n}=\left\{t_{i}\right\}_{i=0}^{n}$, we denote a partition $a=t_{0}<t_{2}<\cdots<t_{n}=b$ of the interval $[a, b]$. For every function 
$f:[0, T] \rightarrow E$ and $1 \leq p<\infty$, we define its Young $p$-variation on $[a, b]$ by the formula

$$
\operatorname{Var}_{p}(f,[a, b])=\sup _{\Pi} \sum_{i=1}^{n}\left(d\left(f\left(t_{i-1}\right), f\left(t_{i}\right)\right)^{p}\right.
$$

and a Riesz $p$-variation on $[a, b]$ by the formula

$$
V_{p}(f,[a, b])=\sup _{\Pi} \sum_{i=1}^{n} \frac{\left(d\left(f\left(t_{i-1}\right), f\left(t_{i}\right)\right)^{p}\right.}{\left(t_{i}-t_{i-1}\right)^{p-1}} .
$$

We denote $\operatorname{Var}_{p}(f,[0, T])$ by $\operatorname{Var}_{p}(f)$ and $V_{p}(f,[0, T])$ by $V_{p}(f)$, respectively. If $\operatorname{Var}_{p}(f)<\infty$ (resp., $V_{p}(f)<\infty$ ), we call $f$ a bounded Young (resp., Riesz) $p$-variation function. The class of all functions of bounded $p$-variations will be denoted by $B \operatorname{Var}_{p}([0, T], E)$ or $B V_{p}([0, T], E)$, respectively. In the sequel, we denote spaces $B \operatorname{Var}_{p}([0, T], E)$ and $B V_{p}([0, T], E)$ simply by $B \operatorname{Var}_{p}(E)$ and $B V_{p}(E)$, respectively. If $\left(X,\|\cdot\|_{X}\right)$ is a Banach space, then $B \operatorname{Var}_{p}(X)$ or $B V_{p}(X)$ with norms $\|f\|_{\operatorname{Var}_{p}}=\sup _{t \in[0, T]}\|f(t)\|_{X}+\left(\operatorname{Var}_{p}(f)\right)^{1 / p}$ and $\|f\|_{V_{p}}=\sup _{t \in[0, T]}\|f(t)\|_{X}+$ $\left(V_{p}(f)\right)^{1 / p}$, respectively, are Banach spaces. For $X=R^{d}$ and considered with the Euclidean norm, we will use the notation $\|x\|$ instead of $\|x\|_{R^{d}}$.

We collect some properties of functions of bounded $V_{p}$-variation in the following proposition.

Proposition $1[10,11]$ Let $f:[0, T] \rightarrow$ E. Then, for every $1 \leq p<\infty$, the following conditions hold:

(a) For every $[a, b] \subset[0, T]$ and $a \leq t \leq b$ we have

$$
V_{p}(f,[a, t])+V_{p}(f,[t, b])=V_{p}(f,[a, b]) .
$$

(b) if $f \in B V_{p}(E)$, then $V_{1}(f,[a, b]) \leq(b-a)^{1-1 / p}\left(V_{p}(f,[a, b])\right)^{1 / p}$ for every $[a, b] \subset[0, T]$, (Jensen inequality).

(c) if $\left(f_{n}\right)$ is a sequence such that $\lim _{n \rightarrow \infty} d\left(f_{n}(t), f(t)\right)=0$ for every $t \in[a, b]$, then $V_{p}(f,[a, b]) \leq \liminf _{n \rightarrow \infty} V_{p}\left(f_{n},[a, b]\right)$.

(d) if $X$ is a reflexive Banach space and $f \in B V_{p}(X)$, then $f$ admits a strong derivative $f^{\prime}$ and $V_{p}(f,[a, b])=\int_{a}^{b}\left\|f^{\prime}(t)\right\|_{X}^{p} \mathrm{~d} t$, (Riesz theorem).

Let $(X,\|\cdot\|)$ be a Banach space, and let $\Pi_{m}: 0=t_{0}<t_{1}<\cdots<t_{m}=T$ be a partition of the interval $[0, T]$. Given a set-valued function $F:[0, T] \rightarrow \operatorname{Comp}(X)$, we set

$$
V_{p}\left(F, \Pi_{m}\right):=\sum_{i=1}^{m} \frac{H_{X}^{p}\left(F\left(t_{i}\right), F\left(t_{i-1}\right)\right)}{\left(t_{i}-t_{i-1}\right)^{p-1}} .
$$


Then, by a Riesz $p$-variation on $[0, T]$, we mean the quantity

$$
V_{p}(F):=\sup _{\Pi_{m}} V_{p}\left(F, \Pi_{m}\right)
$$

By $B V_{p}(\operatorname{Comp}(X))$, we denote the space of all set-valued functions from $[0, T]$ into $\operatorname{Comp}(X)$ having finite Riesz $p$-variation.

\section{Selections of Finite $p$-Variation Set-Valued Functions}

Let $T>0$ be given and let $F:[0, T] \rightarrow \operatorname{Comp}(X)$ be a measurable set-valued function. A measurable function $f:[0, T] \rightarrow X$ is called a measurable selection of $F$ if $f(t) \in F(t)$ for all $t \in[0, T]$. For $1 \leq p<\infty$, define the set

$$
S_{L^{p}}(F)=\left\{f \in L^{p}([0, T], X): f(t) \in F(t) \text { a.e. } \mathrm{t} \in[0, \mathrm{~T}]\right\} .
$$

$S_{L^{p}}(F)$ is a closed subset of $L^{p}([0, T], X)$. It is nonempty if $F$ is $p$-integrably bounded, i.e., if there exists $g \in L^{p}\left([0, T]\right.$ such that $\|F(t)\|_{X} \leq g(t)$ for a.e. $t \in[0, T]$. In such a case, there exists a sequence $\left(f_{n}\right) \subset S_{L^{p}}(F)$ such that $F(t)=\overline{\left\{f_{n}(t)\right\}_{n=1}^{\infty}}$ for all $t \in[0, T]$. The sequence $\left(f_{n}\right)$ is called an $L^{p}$-Castaing representation for $F$. For other properties of measurable set-valued functions and their measurable selections, see, e.g., [5].

Definition 1 Let $F:[0, T] \rightarrow \operatorname{Comp}\left(R^{d}\right)$ be a set-valued function. For $1 \leq p<\infty$, define

$$
S_{V_{p}}(F):=\left\{f \in B V_{p}\left(R^{d}\right): f(t) \in F(t), t \in[0, T]\right\}
$$

the set of selections of $F$ with a bounded Riesz $p$-variation.

Let $F \in \mathcal{C}^{\beta}\left(\operatorname{Comp}\left(R^{d}\right)\right)$. Such set-valued functions need not admit any Hölder or even continuous selection, see, e.g., [10]. However, considering the smaller class $B V_{p}\left(\operatorname{Comp}\left(R^{d}\right)\right) \subset \mathcal{C}^{\beta}\left(\operatorname{Comp}\left(R^{d}\right)\right)$, the following selection theorem holds true.

Proposition 2 [11] Let $F:[0, T] \rightarrow \operatorname{Comp}\left(R^{d}\right)$ be a set-valued function. If $F \in$ $B V_{p}\left(\operatorname{Comp}\left(R^{d}\right)\right)$ for some $1 \leq p<\infty$, then there exist a function $\phi \in B V_{p}\left(R^{d}\right)$ and a sequence of equi-Lipschitzian functions $\left(g_{n}\right)_{n=1}^{\infty}$ with Lipschitz constants $L_{n} \leq 1$ such that taking $f_{n}:=g_{n} \circ \phi$, we have $V_{p}\left(f_{n},[a, b]\right) \leq V_{p}(F,[a, b])$ for every $0 \leq a<b \leq T$ and $F(t)=\overline{\left\{f_{n}(t)\right\}_{n=1}^{\infty}}$ for every $t \in[0, T]$. The set $\left\{f_{n}\right\}_{n=1}^{\infty}$ is a $V_{p}$-Castaing representation for $F$.

Let us note that the set $S_{V_{p}}(F)$ need not be closed in the topology of point convergence even if $F$ is bounded.

Example 1 The set $S_{V_{p}}(F)$ need not be closed in the topology of point convergence even if $F$ is bounded. To see this, let $W$ be a Wiener process defined on some adequate probability space $(\Omega, \mathcal{F}, P)$. Let $W(\cdot, \bar{\omega})$ denote its trajectory connected with a fixed 
$\bar{\omega} \in \Omega$. Then, $M=\sup _{t \in[0, T]}|W(t, \bar{\omega})|<\infty$, because of continuity of trajectories of a Wiener process. Let $F:[0, T] \rightarrow \operatorname{Comp}\left(R^{1}\right)$ be a set-valued function defined by formula $F(t)=[-M, M]$ for every $t \in[0, T]$. Let $\left(\Pi_{n}\right)_{n=1}^{\infty}=\left(\left\{t_{i}\right\}_{i=1}^{n}\right)_{n=1}^{\infty}$ denote a sequence of normal partitions $0=t_{1}<t_{2}<\cdots<t_{n}=T$ of the interval $[0, T]$, and let $W_{n}(\cdot, \bar{\omega})$ denote regularizations of $W(\cdot, \bar{\omega})$ defined by the formula below

$$
W_{n}(t, \bar{\omega})=\left\{\begin{array}{l}
W\left(t_{i}, \bar{\omega}\right) \text { for } t=t_{i} \\
\text { is linear for } t \in\left(t_{i}, t_{i+1}\right)
\end{array} .\right.
$$

It is clear that $W_{n}(t, \bar{\omega}) \in F(t)$. Moreover, for a linear function $g(t)=a t+b$, we have $V_{p}\left(g,\left[t_{i}, t_{i+1}\right]\right)=|a|^{p}\left(t_{i+1}-t_{i}\right)<\infty$. Therefore, we get by Proposition $1(\mathrm{a})$,

$$
\begin{aligned}
& V_{p}\left(W_{n}(\cdot, \bar{\omega}),[0, T]\right)=\sum_{i=1}^{n-1} V_{p}\left(W_{n}(\cdot, \bar{\omega}),\left[t_{i}, t_{i+1}\right]\right) \\
& \quad \leq \max \left\{\frac{\left|W\left(t_{i+1}, \bar{\omega}\right)-W\left(t_{i}, \bar{\omega}\right)\right|^{p}}{\left(t_{i+1}-t_{i}\right)^{p}}, i=1,2, \ldots, n-1\right\} \cdot \sum_{i=1}^{n-1}\left(t_{i+1}-t_{i}\right)<\infty .
\end{aligned}
$$

It means that $W_{n}(\cdot, \bar{\omega}) \in S_{V_{p}}(F)$. But $W_{n}(t, \bar{\omega})$ tends to $W(t, \bar{\omega})$ for every $t \in[0, T]$. Since $V_{p}\left(W(\cdot, \bar{\omega})=+\infty\right.$ for every $1 \leq p<2$, then $W(\cdot, \bar{\omega}) \notin S_{V_{p}}(F)$.

However, the set $S_{V_{p}}(F)$ is closed in the norm $\|\cdot\|_{V_{p}}$ because of Jensen inequality $\left\|f_{n}(t)-f(t)\right\| \leq \max \left\{1, T^{1-1 / p}\right\}\left\|f_{n}-f\right\|_{V_{p}} \rightarrow 0$ and Proposition 1(c).

Proposition 3 Let $F:[0, T] \rightarrow \operatorname{Comp}\left(R^{d}\right)$ be a set-valued function, $F \in$ $B V_{p}\left(\operatorname{Comp}\left(R^{d}\right)\right)$ for some $1 \leq p<\infty$. Let $\left\{f_{m}\right\}_{m=1}^{\infty}$ be the $V_{p}$-Castaing representation of $F$ given in Proposition 2. Then, for every $f \in S_{V_{p}}(F)$ and every $\epsilon>0$, there exist a finite measurable covering $A_{1}, \ldots, A_{n}$ of the interval $[0, T]$ and functions $f_{k_{1}}, \ldots, f_{k_{n}} \in\left\{f_{m}\right\}_{m=1}^{\infty}$ such that

$$
\left\|f-\sum_{j=1}^{n} \mathbb{I}_{A_{j}} \cdot f_{k_{j}}\right\|_{L^{p}}<\epsilon .
$$

Moreover, for every $f \in S_{V_{p}}(F)$ and every $\epsilon>0$, there exist $n \geq 1$, a partition $\Pi_{n}: 0=t_{0}<t_{1}<\cdots<t_{n}=T$ and functions $f_{k_{0}}, \ldots, f_{k_{n}} \in\left\{f_{m}\right\}_{m=1}^{\infty}$ such that

$$
\left\|f-\sum_{j=0}^{n-1} \mathbb{I}_{\left[t_{j}, t_{j+1}\right)} \cdot f_{k_{j}}\right\|_{\infty}<\epsilon .
$$

Proof Since $S_{V_{p}}(F) \subset S_{L^{p}}(F)$ and the $V_{p}$-Castaing representation of $F$ is also an $L^{p}$-Castaing representation of $F$ introduced in [9], then the proof follows by Lemma 1.3 of [20]. 
We prove second inequality. Let $f \in S_{V_{p}}(F)$ be arbitrary taken. There exists $\delta$ such that $\|f(t)-f(s)\|<\epsilon / 3$ and $\left\|f_{m}(t)-f_{m}(s)\right\|<\epsilon / 3$ for every $|t-s|<\delta$ (see Proposition 1). Let us take a partition $\Pi_{n}: 0<\delta<2 \delta<\cdots<n \delta<T$. Since $f(t) \in\left\{f_{m}(t)\right\}_{m=1}^{\infty}$, then for every $k=0,1, \ldots, n$ there exists $m_{k}$ such that $\| f(k \delta)-$ $f_{m_{k}}(k \delta) \|<\epsilon / 3$. Therefore, $\left\|f(t)-f_{m_{k}}(t)\right\|<\epsilon$ for $t \in[k \delta, \min \{(k+1) \delta, T\}]$.

Thus,

$$
\left\|f-\sum_{k=0}^{n-1} \mathbb{I}_{\left[t_{k}, t_{k+1}\right)} \cdot f_{m_{k}}\right\|_{\infty}=\left\|f-\sum_{k=0}^{n-1} \mathbb{I}_{[k \delta, \min \{(k+1) \delta, T\}]} \cdot f_{m_{k}}\right\|_{\infty} \leq \epsilon
$$

Let us note that a similar approximation property with respect to $V_{p}$-variation norm need not hold true.

Now we introduce the notion of $V_{p}$-decomposable selections of set-valued functions and investigate their properties.

Let $(\Omega, \mathcal{A}, \mu)$ be a measure space. A set $\Lambda \subset L^{p}\left(\Omega, \mathcal{A}, \mathbb{R}^{d}\right)$ is said to be $L^{p}$ decomposable, if for every $f_{1}, f_{2} \in \Lambda$ and every $A \in \mathcal{A}$ one has $\mathbb{I}_{A} \cdot f_{1}+\mathbb{I}_{A^{\sim}} \cdot f_{2} \in \Lambda$, where $A^{\sim}$ denotes the complement of the set $A$ in $\Omega$. For any $L^{p}$-decomposable sets $\mathcal{H}, \mathcal{K} \subset L^{p}\left(\Omega, \mathcal{A}, \mathbb{R}^{d}\right)$, the Minkowski sum $\mathcal{H}+\mathcal{K}$ is again an $L^{p}$-decomposable subset of the space $L^{p}\left(\Omega, \mathcal{A}, \mathbb{R}^{d}\right)$.

For a given set $B \subset L^{p}\left(\Omega, \mathcal{A}, \mathbb{R}^{d}\right)$, we denote the set $\left\{\sum_{k=1}^{n} \mathbb{I}_{A_{k}} \cdot \beta_{k}: A_{k} \in\right.$ $\left.\mathcal{A}, \beta_{k} \in B, n=1,2, \ldots\right\}$ by $\operatorname{dec}_{L^{p}}(B)$ and call it an $L^{p}$-decomposable hull of a set $B$.

By $\overline{\operatorname{dec}}_{L^{p}}(B)$, we denote a closed $L^{p}$-decomposable hull of a set $B$. Similarly as in the case of convex and closed convex hulls, they are the smallest $L^{p}$-decomposable and closed $L^{p}$-decomposable sets containing the set $B$, respectively.

From this, it follows that the set $S_{L^{p}}(F)$ consisting of all $L^{p}$-selectors of a given measurable set-valued function $F$ is always $L^{p}$-decomposable and therefore, $S_{L^{p}}(F)=\overline{\operatorname{dec}}_{L^{p}}\left(S_{L^{p}}(F)\right)$. Conversely, if a closed set $\Lambda \subset L^{p}\left(\Omega, \mathcal{A}, \mathbb{R}^{d}\right)$ is $L^{p}-$ decomposable, then there exists a measurable set-valued function $F: \Omega \rightarrow R^{d}$ such that $\Lambda=S_{L^{p}}(F)$, (see [20]). For other properties of $L^{p}$-decomposable sets, see [19].

$L^{1}$-decomposability of the set of $L^{1}$-selectors of a given measurable set-valued function $F$ is crucial for investigating properties of a set-valued Aumann integral of $F$ defined by the formula

$$
\int_{A} F(t) \mathrm{d} \mu=\left\{\int_{A} f(t) \mathrm{d} \mu: f \in S_{L^{1}}(F)\right\} .
$$

Unfortunately, the set $S_{V_{p}}(F)$ need not be $L^{p}$-decomposable for any $p \geq 1$, and therefore, if one defines a set-valued Young integral in the Aumann's sense, it is difficult to obtain its reasonable properties. This leads to the idea of a different type of decomposability called $V_{p}$-decomposability.

It follows from Proposition 1(d) that a function $f$ belongs to $B V_{p}\left(R^{d}\right)$ if and only if its strong derivative $f^{\prime}$ belongs to $L^{p}([0, T]), f(t)=f(0)+\int_{0}^{t} f^{\prime}(s) \mathrm{d} s$ and 
$V_{p}(f,[0, t])=\int_{0}^{t}\left\|f^{\prime}(s)\right\|^{p} \mathrm{~d} s$ for every $t \in[0, T]$. This property has been inspiring to the following definition.

Definition 2 A set $\Lambda \subset B V_{p}\left(R^{d}\right)$ is $V_{p}$-decomposable (decomposable in the sense of its Riesz $p$-variation) if for every $f_{1}, f_{2} \in \Lambda$ and every $a \in[0, T]$ the function $f=f_{1} \oplus_{a} f_{2}$ defined by

$$
f(t)=f_{1}(0)+\int_{0}^{t}\left(\mathbb{I}_{[0, a)}(s) \cdot f_{1}^{\prime}(s)+\mathbb{I}_{[a, T]}(s) \cdot f_{2}^{\prime}(s)\right) \mathrm{d} s
$$

belongs to the set $\Lambda$.

For a given set $B \subset B V_{p}\left(R^{d}\right)$ by $\operatorname{dec}_{V_{p}}(B)$, we denote a $V_{p}$-decomposable hull of a set $B$, i.e., the smallest $V_{p}$-decomposable set containing the set $B$.

Remark 1 Every function $f=f_{1} \oplus_{a} f_{2}$ from Definition 2 can be represented by the formula

$$
f(t)= \begin{cases}f_{1}(t) & \text { for } 0 \leq t<a \\ f_{2}(t)-f_{2}(a)+f_{1}(a) & \text { for } \quad a \leq t \leq T\end{cases}
$$

Moreover, for every $B \subset B V_{p}\left(\left(R^{d}\right)\right.$, we have

$$
\operatorname{dec}_{V_{p}}(B)=\left\{f \in B V_{p}\left(R^{d}\right): f(t)=f_{1}(0)+\int_{0}^{t}\left(\sum_{i=0}^{m-1} \mathbb{I}_{\left[t_{i}, t_{i+1}\right)}(s) \cdot f_{i}^{\prime}(s)\right) \mathrm{d} s:\right.
$$

$\left.\Pi_{m}: 0=t_{0}<\cdots<t_{m}=T, m=1,2, \ldots, \quad f_{i} \in B, i=1, \ldots, m\right\}$.

Definition 3 A set $\mathcal{R} \subset B V_{p}\left(R^{d}\right)$ is called an integral if there exist $x_{0} \in R^{d}$ and a measurable and $p$-integrably bounded set-valued function $\Phi:[0, T] \rightarrow \operatorname{Conv}\left(R^{d}\right)$ such that

$$
\mathcal{R}=x_{0}+\int \Phi(s) \mathrm{d} s=\left\{f \in B V_{p}\left(R^{d}\right): f(\cdot)=x_{0}+\int_{0}^{\cdot} \phi(s) \mathrm{d} s, \phi \in S_{L^{p}}(\Phi)\right\} .
$$

We denote by $\mathcal{R}(t)$ the set

$$
\mathcal{R}(t)=\{f(t): f(\cdot) \in \mathcal{R}\}=\left\{x_{0}+\int_{0}^{t} \phi(s) \mathrm{d} s, \phi \in S_{L^{p}}(\Phi)\right\}
$$

Theorem 1 Let $\mathcal{R} \subset B V_{p}\left(R^{d}\right)$ be an integral. Then, $\mathcal{R}$ is closed with respect to the norm $\|\cdot\|_{\infty}$ and $V_{p}$-decomposable.

Proof If $\mathcal{R}$ is an integral, then for every $t \in[0, T] \mathcal{R}(t)$ is a closed subset of $R^{d}$ by Theorem 8.6.7 of [5]. Let $\left(f_{n}\right)_{n=1}^{\infty} \subset \mathcal{R}$ be a sequence convergent to some $f$ with respect to the norm $\|\cdot\|_{\infty}$. Since $\mathcal{R}$ is an integral, then $f_{n}(t)=x_{0}+\int_{0}^{t} \phi_{n}(s) \mathrm{d} s$ for 
some $\phi_{n} \in S_{L^{p}}(\Phi)$. But $f_{n}(0)=x_{0}$ and therefore, $f(0)=x_{0}$. Moreover, since $\Phi$ is $p$-integrably bounded by some function $g \in L^{p}([0, T])$, then $\sup _{n} V_{p}\left(f_{n}\right) \leq\|g\|_{L^{p}}$. It follows from Proposition 1(c) that $V_{p}(f) \leq\|g\|_{L^{p}}$. Therefore, $f \in B V_{p}\left(R^{d}\right)$ and $f(t)=x_{0}+\int_{0}^{t} f^{\prime}(s) \mathrm{d} s$. Since $\Phi$ is $p$-integrably bounded and has closed and bounded values, then the set $S_{L^{p}}(\Phi)$ is closed, bounded and convex in $L^{p}([0, T])$. Therefore, it is weakly compact there. Thus, there exists a subsequence $\left(\phi_{n_{k}}\right)$ of $\left(\phi_{n}\right)$ weakly convergent to some $\phi \in S_{L^{p}}(\Phi)$. Let $J: L^{p}([0, T]) \rightarrow C([0, T])$ be a linear operator defined by formula $J(\psi)=x_{0}+\int_{0}^{\cdot} \psi(s) \mathrm{d} s$. Since $J$ is norm-to-norm continuous, then it is also weak-to-weak continuous. Thus, $f_{n_{k}}=x_{0}+\int_{0}^{\cdot} \phi_{n_{k}}(s) \mathrm{d} s$ tends weakly to $x_{0}+\int_{0}^{\cdot} \phi(s) \mathrm{d} s$. But $\left(f_{n_{k}}\right)$ tends to $f=x_{0}+\int_{0}^{\cdot} f^{\prime}(s) \mathrm{d} s$ in $\|\cdot\|_{\infty}$ norm. Thus, $\phi=f^{\prime}$, and therefore, $f^{\prime} \in S_{L^{p}}(\Phi)$. This implies $f \in \mathcal{R}$, which proves the closedness of $\mathcal{R}$.

Now let us take $f_{1}, f_{2} \in \mathcal{R}$. There exist a set-valued function $\Phi$ and functions $\phi_{1}, \phi_{2} \in S_{L^{p}}(\Phi)$ such that $f_{1}(t)=x_{0}+\int_{0}^{t} \phi_{1}(s) \mathrm{d} s$ and $f_{2}(t)=x_{0}+\int_{0}^{t} \phi_{2}(s) \mathrm{d} s$ for every $t \in[0, T]$. Let $a \in[0, T]$ be arbitrarily taken and let $\gamma(t)=\mathbb{I}_{[0, a)}(s) \cdot \phi_{1}(s)+$ $\mathbb{I}_{[a, T]}(s) \cdot \phi_{2}(s)$. Then $\gamma \in S_{L^{p}}(\Phi)$ and therefore, $f=f_{1} \oplus_{a} f_{2}=x_{0}+\int \gamma(s) \mathrm{d} s \in \mathcal{R}$. It means that $\mathcal{R}$ is $V_{p}$-decomposable.

Theorem 2 Let $\mathcal{R} \subset B V_{p}\left(R^{d}\right), \mathcal{R}(0)=x_{0}$, be bounded, $V_{p}$-decomposable, convex and closed with respect to the norm $\|\cdot\|_{\infty}$. Then, $\mathcal{R}$ is an integral.

Proof Assuming that $\mathcal{R} \subset B V_{p}\left(R^{d}\right)$, let $f_{1}, f_{2} \in \mathcal{R}$ and $a \in[0, T]$ be arbitrarily taken. If $f=f_{1} \oplus_{a} f_{2}$, then $f \in \mathcal{R}$ by the assumption of $V_{p}$-decomposability. We define the set $M$ by the formula

$$
M=\left\{\phi \in L^{p}([0, T]): x_{0}+\int \phi(s) \mathrm{d} s \in \mathcal{R}\right\} .
$$

Then, $M$ is convex in $L^{p}([0, T])$. It is bounded and closed in $L^{p}([0, T])$ by Proposition $1(\mathrm{~d})$.

Since $\mathcal{R}(0)=x_{0}$, then $\mathcal{R}=\left\{f_{\alpha}: f_{\alpha}=x_{0}+\int f_{\alpha}^{\prime}(s) \mathrm{d} s ; f_{\alpha}^{\prime} \in M\right\}$. We will show that the set $M$ is $L^{p}$-decomposable in $L^{p}([0, T], \beta([0, T]), \lambda)$, i.e., we will show that for every set $A \in \beta([0, T])$ and any $\phi, \psi \in M$, the function $\gamma=\mathbb{I}_{A} \cdot \phi+\mathbb{I}_{A^{\sim}} \cdot \psi$ belongs to the set $M . \beta([0, T])$, as usual, denotes here the Borel $\sigma$ algebra of subsets of the interval $[0, T]$, and $\lambda$ is a Lebesgue measure.

We take a partition $\Pi_{n}: 0=t_{0}<t_{1}<\cdots<t_{2 n}<t_{2 n+1}=T$ and the set $A$ of the form $A=\bigcup_{i=0}^{n}\left[t_{2 i}, t_{2 i+1}\right)$. Since $\mathcal{R}$ is $V_{p}$-decomposable, it is easy to see that taking any $f_{1}, \ldots, f_{2 n+1} \in \mathcal{R}$ a function $f$ given by the formula $f(t)=$ $x_{0}+\int_{0}^{t} \sum_{i=0}^{2 n} \mathbb{I}_{\left[t_{i}, t_{i+1}\right)}(s) \cdot f_{i+1}^{\prime}(s) \mathrm{d} s$ belongs to $\mathcal{R}$. Therefore, taking $f_{2 i}^{\prime}=\phi$ for $i=1,2, \ldots n$ and $f_{2 i+1}^{\prime}=\psi$ for $i=0,1, \ldots n$, we have

$$
\begin{aligned}
x_{0}+\int \gamma(s) \mathrm{d} s & =x_{0}+\int\left(\mathbb{I}_{A}(s) \cdot \phi(s)+\mathbb{I}_{A^{\sim}}(s) \cdot \psi(s)\right) \mathrm{d} s \\
& =x_{0}+\int_{0}^{t} f^{\prime}(s) \mathrm{d} s \in \mathcal{R} .
\end{aligned}
$$

It means that $\gamma \in M$. 
Let $\mathcal{M}=\bigcup_{n=1}^{\infty} \bigcup_{\Pi_{n}}\left\{B \subset[0, T]: B=\bigcup_{i=0}^{n-1}\left[t_{2 i}, t_{2 i+1}\right)\right\}$. Then, $\mathcal{M}$ is a ring generating a $\sigma$-algebra $\beta([0, T])$. We will show that $\mathcal{M}$ is a monotone class also. To this end, assume that $\left(A_{i}\right)_{i=1}^{\infty} \subset \mathcal{M}$ and $A_{i} \subset A_{i+1}$. We prove that the set $A=\bigcup_{i=1}^{\infty} A_{i}$ belongs to $\mathcal{M}$. We can find an infinite partition $\Pi_{\infty}: 0=t_{0}<t_{1}<t_{2}<\cdots$ of $[0, T]$, and taking $\tilde{A_{k}}=\bigcup_{i=0}^{k}\left[t_{2 i}, t_{2 i+1}\right)$, we get $\tilde{A}_{k} \subset \tilde{A}_{k+1} \subset A$ and $A=\lim _{k \rightarrow \infty} \tilde{A_{k}}=$ $\bigcup_{k=1}^{\infty} \tilde{A}_{k}$. Therefore, $\mathbb{I}_{A}(s)=\lim _{k \rightarrow \infty} \mathbb{I}_{\tilde{A}_{k}}(s)$ for every $s \in[0, T]$. Since the sets $\left(\tilde{A_{k}}\right) \sim$ form a decreasing family, then a sequence $\left(\mathbb{I}_{\left(\tilde{A}_{k}\right)} \sim(s)\right)$ is a decreasing sequence of functions convergent to $\mathbb{I}_{A^{\sim}}(s)$, where $A^{\sim}=\bigcap_{k=1}^{\infty}\left(\tilde{A}_{k}\right)^{\sim}$.

Let us take any $\phi, \psi \in M, A=\bigcup_{i=0}^{\infty}\left[t_{2 i}, t_{2 i+1}\right)$, and let $\gamma(s)=\mathbb{I}_{A}(s) \cdot \phi(s)+$ $\mathbb{I}_{A^{\sim}}(s) \cdot \psi(s)$. Then, $\gamma(s)=\lim _{k \rightarrow \infty} \gamma_{k}(s)$, where $\gamma_{k}(s)=\mathbb{I}_{\tilde{A}_{k}}(s) \cdot \phi(s)+\mathbb{I}_{\left(\tilde{A_{k}}\right)} \sim(s)$. $\psi(s)$. It was shown in the first part of the proof that $\gamma_{k}(s) \in M$, because of $x_{0}+$ $\int \gamma_{k}(s) \mathrm{d} s \in \mathcal{R}$. We show that $\gamma \in M$, i.e., that $f=x_{0}+\int \gamma(s) \mathrm{d} s \in \mathcal{R}$. We know that $f_{k}=x_{0}+\int \gamma_{k}(s) \mathrm{d} s \in \mathcal{R}$. We have

$$
\left\|f_{k}-f\right\|_{\infty}=\sup _{t \in[0, T]}\left\|\int_{0}^{t}\left(\gamma_{k}(s)-\gamma(s)\right) \mathrm{d} s\right\| \leq \int_{0}^{T}\left\|\gamma_{k}(s)-\gamma(s)\right\| \mathrm{d} s .
$$

However, $\gamma_{k}(s) \rightarrow \gamma(s)$ a.e. and the sequence $\left\|\gamma_{k}(s)-\gamma(s)\right\|$ admits a $p$-integrable majorant $2|\phi(s)|+2|\psi(s)|$. Therefore, $\left\|f_{k}-f\right\|_{\infty} \rightarrow 0$. Since $\mathcal{R}$ is closed by the assumption, then $f \in \mathcal{R}$ and therefore, $\gamma \in M$.

We have shown that the set

$$
W=\left\{A \in \beta([0, T]): \mathbb{I}_{A} \cdot \phi+\mathbb{I}_{A^{\sim}} \cdot \psi \in M \text { if } \phi, \psi \in M\right\}
$$

contains a ring generating $\beta([0, T])$ and a monotone class

$$
\Lambda=\bigcup_{\Pi_{\infty}}\left\{A, A^{\sim} \subset[0, T]: A=\bigcup_{i=0}^{\infty}\left[t_{2 i}, t_{2 i+1}\right)\right\} .
$$

From the monotone class theorem, we deduce that for every $\phi, \psi \in M$ and every set $Q \in \beta([0, T])$ the set $\mathbb{I}_{Q} \phi+\mathbb{I}_{Q \sim} \psi$ belongs to $M$. Therefore, $M$ is $L^{p}$-decomposable and by Theorem 3.1 of [20] there exists a measurable set-valued function $\Phi:[0, T] \rightarrow$ $C l\left(R^{d}\right)$ such that $S_{L^{p}}(\Phi)=M=\left\{\phi \in L^{p}([0, T]): x_{0}+\int \phi(s) \mathrm{d} s\right\} \in \mathcal{R}$. It means that $\mathcal{R}=x_{0}+\int \Phi(s) \mathrm{d} s$. Since $S_{L^{p}}(\Phi)=M$ is convex, then $\Phi$ has convex values by Theorem 1.5 from [20]. Moreover, $\Phi$ is $p$-integrably bounded by the boundedness of $M$. Therefore, $\mathcal{R}$ should be an integral.

Definition 4 Let $X$ be a real normed linear space. Let $A, B \in \operatorname{Conv}(X)$. The set $C \in$ $\operatorname{Conv}(X)$ is said to be the Hukuhara difference $A \div B$ if $A=B+C$. Consider a setvalued mapping $G: R^{1} \rightarrow \operatorname{Conv}(X)$. We say that $G$ admits a Hukuhara differential at $t_{0} \in R^{1}$, if there exists a set $D_{H} G\left(t_{0}\right) \in \operatorname{Conv}(X)$ and such that the limits

$$
\lim _{\Delta t \rightarrow 0+} \frac{G\left(t_{0}+\Delta t\right) \div G\left(t_{0}\right)}{\Delta t}
$$


and

$$
\lim _{\Delta t \rightarrow 0+} \frac{G\left(t_{0}\right) \div G\left(t_{0}-\Delta t\right)}{\Delta t}
$$

exist and are equal to the set $D_{H} G\left(t_{0}\right)$.

For a detailed discussion of the properties and applications of the Hukuhara differentiable multifunctions, we refer the reader to [23].

Now we are ready to prove the main decomposability results of the section.

Theorem 3 If a closed and bounded set $\mathcal{R} \subset B V_{p}\left(R^{d}\right)$ with $\mathcal{R}(0)=x_{0}$ is $V_{p^{-}}$ decomposable, then there exists a measurable and p-integrably bounded set-valued function $\Phi:[0, T] \rightarrow \operatorname{Comp}\left(R^{d}\right)$ such that the set-valued function $t \rightarrow \mathcal{R}(t)$ is Hukuhara differentiable for almost every $t \in[0, T]$ and $D_{H} \mathcal{R}(t)=\overline{c o} \Phi(t)$.

Proof Assume that a closed and bounded set $\mathcal{R}$ in $B V_{p}\left(R^{d}\right)$ is $V_{p}$-decomposable. It is also closed with respect to $\|\cdot\|_{\infty}$. Therefore, it follows by Theorem 2 that $\mathcal{R}$ is an integral, i.e., there exists a measurable and a $p$-integrably bounded set-valued function $\Phi:[0, T] \rightarrow \operatorname{Comp}\left(R^{d}\right)$ such that

$$
\mathcal{R}=x_{0}+\int \Phi(s) \mathrm{d} s=\left\{f \in B V_{p}\left(R^{d}\right): f(\cdot)=x_{0}+\int_{0}^{\cdot} \phi(s) \mathrm{d} s, \phi \in S_{L^{p}}(\Phi)\right\} .
$$

Since $\mathcal{R}(0)=x_{0}$, then $\mathcal{R}(t)$ is an Aumann integral, $\mathcal{R}(t)=x_{0}+\int_{0}^{t} \Phi(s) \mathrm{d} s=$ $\left\{f(t)=x_{0}+\int_{0}^{t} \phi(s) \mathrm{d} s, \phi \in S_{L^{p}}(\Phi)\right\}$. From this, we deduce that the Hukuhara derivative $D_{H}(\mathcal{R}(t))$ exists for almost every $t \in[0, T]$ and $D_{H} \mathcal{R}(t)=\overline{c o} \Phi(t)$, see, e.g., [29].

Remark 2 If a set $\mathcal{R} \subset B V_{p}\left(R^{d}\right)$ is an integral, then $\mathcal{R}(t)=x_{0}+\int_{0}^{t} \Phi(s) \mathrm{d} s$ for every $t \in[0, T]$ and some measurable and $p$-integrably bounded set-valued function $\Phi$. The reverse implication need not hold as the following example shows.

Example 2 Let $\Phi:[0,1] \rightarrow R^{1}$ be a constant set-valued function $\Phi(t) \equiv[0,1]$. Let $\mathcal{R}(t)=\int_{0}^{t} \Phi(s) \mathrm{d} s=[0, t]$. Then, $\mathcal{R}(\cdot)$ is Hukuhara differentiable with $D_{H}(\mathcal{R}(\cdot))(t)=\Phi(t)$. We will show that $\mathcal{R}=S_{V_{p}}(\mathcal{R}(\cdot))$ is not an integral. Let us take $f_{1}(t) \equiv 0$ and

$$
f_{2}(t)=\left\{\begin{array}{cc}
t & \text { for } 0 \leq t<1 / 2 \\
-t+1 & \text { for } 1 / 2 \leq t \leq 1
\end{array}\right.
$$

Of course, $f_{1}, f_{2} \in S_{V_{p}}(\mathcal{R}(\cdot))$. However,

$$
f(t)=\left(f_{1} \oplus_{1 / 2} f_{2}\right)(t)=\left\{\begin{array}{cll}
0 & \text { for } & 0 \leq t<1 / 2 \\
-t+1 / 2 & \text { for } & 1 / 2 \leq t \leq 1
\end{array} .\right.
$$

Then, $f(t) \notin \mathcal{R}(t)$ for $t \in[1 / 2,1]$, and therefore, $f=\left(f_{1} \oplus_{1 / 2} f_{2}\right) \notin S_{V_{p}}(\mathcal{R}(\cdot))=\mathcal{R}$. It means that $\mathcal{R}$ is not an integral. 
Theorem 4 Let $F:[0, T] \rightarrow \operatorname{Conv}\left(R^{d}\right)$ be a Hukuhara differentiable set-valued function, $F \in B V_{p}\left(\operatorname{Conv}\left(R^{d}\right)\right), F(0)=x_{0}$. Then, the set

$$
\mathcal{I S}(F)=\left\{f \in B V_{p}\left(R^{d}\right): f \in S_{V_{p}}(F) \text { and } f^{\prime} \in S_{L^{p}}\left(D_{H}(F)\right)\right\}
$$

is $V_{p}$-decomposable and therefore, it is an integral.

Proof Really, let $f, g \in \mathcal{R}$. Then, $f, g \in S_{V_{p}}(F)$. Therefore, $f^{\prime}, g^{\prime} \in L^{p}([0, T])$ and $f^{\prime}, g^{\prime} \in S_{L^{p}}\left(D_{H}(F)\right)$. Then, the function $\gamma=\mathbb{I}_{[0, a)} \cdot f^{\prime}+\mathbb{I}_{[a, T]} \cdot g^{\prime} \in S_{L^{p}}\left(D_{H}(F)\right)$ because the set $S_{L^{p}}\left(D_{H}(F)\right)$ is $L^{p}$-decomposable. From this, we get $\left(f \oplus_{a} g\right)(t)=$ $x_{0}+\int_{0}^{t} \gamma(s) \mathrm{d} s \in x_{0}+\int_{0}^{t} D_{H}(F)(s) \mathrm{d} s=F(t)$. Since $V_{p}\left(\left(f \oplus_{a} g\right)\right) \leq V_{p}(f)+$ $V_{p}(g)<\infty$, then $\left(f \oplus_{a} g\right) \in S_{V_{p}}(F)$, and therefore, $\left(f \oplus_{a} g\right) \in \mathcal{R}$. We proved that $\mathcal{R}$ is $V_{p}$-decomposable and it is an integral by Theorem 2 .

Let $C \in \operatorname{Conv}\left(R^{d}\right)$ and let $\sigma(\cdot, C): R^{d} \rightarrow R^{1}, \sigma(p, C)=\sup _{y \in C}<p, y>$ be a support function of $C$. Let $\Sigma$ denote the unit sphere in $R^{d}$, and let $V$ denote a Lebesgue measure of a closed unit ball $B(0,1)$ in $R^{d}$, i.e., $V=\pi^{d / 2} / \Gamma\left(1+\frac{d}{2}\right)$ with $\Gamma$ being the Euler function. Let $p_{V}$ be a normalized Lebesgue measure on $B(0,1)$, i.e., $d p_{V}=d p / V$. Let

$$
\begin{gathered}
\mathcal{M}=\{\mu: \mu \text { is a probability measure on } B(0,1) \text { having } \\
\text { the } \left.C^{1}-\text { density } d \mu / d p_{V} \text { with respect to measure } p_{V}\right\} .
\end{gathered}
$$

Let $\xi_{\mu}:=d \mu / d p_{V}$, and let $\nabla \xi_{\mu}$ denote the gradient of $\xi_{\mu}$. By $\omega$, we denote a Lebesgue measure on $\Sigma$. The function $S t_{\mu}: \operatorname{Conv}\left(R^{d}\right) \rightarrow R^{d}$ called a generalized Steiner center, and given by the formula

$$
S t_{\mu}(C)=V^{-1}\left(\int_{\Sigma} p \sigma(p, C) \xi_{\mu}(p) \mathrm{d} \omega(p)-\int_{B(0,1)} \sigma(p, C) \nabla \xi_{\mu}(p) \mathrm{d} p\right)
$$

for every $\mu \in \mathcal{M}$, has the following properties.

For $A, B, C \in \operatorname{Conv}\left(R^{d}\right)$ and $a, b \in R^{1}$

$$
\begin{gathered}
S t_{\mu}(C) \in C, S t_{\mu}(a A+b B)=a S t_{\mu}(A)+b S t_{\mu}(B), \\
\left\|S t_{\mu}(A)-S t_{\mu}(B)\right\| \leq L_{\mu} \cdot H_{R^{d}}(A, B),
\end{gathered}
$$

where $L_{\mu}=d \max _{p \in \Sigma} \xi_{\mu}(p)+\max _{p \in B(0,1)}\left\|\nabla \xi_{\mu}(p)\right\|$ (see e.g., [13]).

Since the set $C_{d}^{1}=\left\{\xi \in C^{1}\left(B(0,1), R^{+}\right): \int_{B} \xi \mathrm{d} p_{V}=1\right\}$ is separable, then there exists a countable subset $\left\{\xi_{n}\right\} \subset C_{d}^{1}$ dense in $C_{d}^{1}$ with respect to supremum norm. Let $\left\{\mu_{n}\right\}$ be a sequence of measures from $\mathcal{M}$ with densities $\left\{\xi_{n}\right\}$. It is known that every set $C \subset \operatorname{Conv}\left(R^{d}\right)$ has a representation

$$
C=\overline{\left\{S t_{\mu}(C)\right\}_{\mu \in \mathcal{M}}},
$$


where $S t_{\mu}(C)$ are generalized Steiner points of $C$ given by formula (4), see also [13]. Therefore, by separability of $C_{d}^{1}$, we have

$$
C=\overline{\left\{S t_{\mu_{n}}(C)\right\}_{n=1}^{\infty}} \text {. }
$$

Let $F:[0, T] \rightarrow \operatorname{Conv}\left(R^{d}\right)$ be a set-valued function. Then,

$$
S t_{\mu}\left(\int_{0}^{t} F(s) \mathrm{d} s\right)=\int_{0}^{t}\left(S t_{\mu}(F(s)) \mathrm{d} s\right.
$$

for every $t \in[0, T]$ by [8] and we obtain

$$
\int_{0}^{t} F(s) \mathrm{d} s=\overline{\left\{S t_{\mu_{n}}\left(\int_{0}^{t} F(s) \mathrm{d} s\right)\right\}_{n=1}^{\infty}}=\overline{\left\{\int_{0}^{t}\left(S t_{\mu_{n}}(F(s)) \mathrm{d} s\right\}_{n=1}^{\infty}\right.} .
$$

Assume that $F \in B V_{p}\left(\operatorname{Conv}\left(R^{d}\right)\right)$ is Hukuhara differentiable, $F(0)=x_{0}$, and consider again a set $\mathcal{I} \mathcal{S}(F)$ defined by (3). This set is an integral by Theorem 4 . We prove the following result.

Theorem 5 Let $F \in B V_{p}\left(\operatorname{Conv}\left(R^{d}\right)\right)$ be a Hukuhara differentiable set-valued function, $F(0)=x_{0}$. Then, there exists a Castaing representation $\left\{f_{n}\right\}_{n=1}^{\infty}$ of $F$ with $f_{n} \in \mathcal{I S}(F)$ for every $n=1,2, \ldots$

Proof Since $F(t)=x_{0}+\int_{0}^{t} D_{H}(F)(s) \mathrm{d} s$, then by formula (7) we obtain

$$
F(t)=x_{0}+\int_{0}^{t} D_{H}(F)(s) \mathrm{d} s=x_{0}+\overline{\left\{\int_{0}^{t} S t_{\mu_{n}}\left(D_{H}(F)(s)\right) \mathrm{d} s\right\}_{n=1}^{\infty}}
$$

It means that the sequence $\left\{f_{n}\right\}_{n=1}^{\infty}$ defined by the formula $f_{n}(t)=x_{0}+$ $\int_{0}^{t} S t_{\mu_{n}}\left(D_{H}(F)(s)\right) \mathrm{d} s$ is a Castaing representation of $F$. Moreover, $f_{n}^{\prime}(t)=$ $S t_{\mu_{n}}\left(D_{H}(F)(t)\right) \in D_{H}(F)(t)$. We have to show that $f_{n} \in B V_{p}\left(R^{d}\right)$ and $f_{n}^{\prime} \in$ $L^{p}([0, T])$. We know that $f_{n}(t)=x_{0}+\int_{0}^{t} S t_{\mu_{n}}\left(D_{H}(F)(s)\right) \mathrm{d} s=x_{0}+S t_{\mu_{n}}(F(t))$ by equality (6). It follows from formula (5) that

$$
\left\|f_{n}(t)-f_{n}(s)\right\| \leq L_{\mu} \cdot H_{R^{d}}(F(t), F(s)),
$$

where $L_{\mu}=d \max _{p \in \Sigma} \xi_{\mu}(p)+\max _{p \in B(0,1)}\left\|\nabla \xi_{\mu}(p)\right\|$. Therefore, for every $0 \leq$ $a<b<\leq T$,

$$
\begin{aligned}
V_{p}\left(f_{n},[a, b]\right) & =\sup _{\Pi_{m}} \sum_{i=0}^{m} \frac{\left\|f_{n}\left(t_{i}\right)-f_{n}\left(t_{i-1}\right)\right\|^{p}}{\left(t_{i}-t_{i-1}\right)^{p-1}} \\
& \leq L_{\mu} \sup _{\Pi_{m}} \sum_{i=0}^{m} \frac{\left(H_{R^{d}}\left(F\left(t_{i-1}\right), F\left(t_{i}\right)\right)\right)^{p}}{\left(t_{i}-t_{i-1}\right)^{p-1}}=L_{\mu} V_{p}(F,[a, b])<\infty .
\end{aligned}
$$


Therefore, $f_{n} \in B V_{p}\left(R^{d}\right)$.

Now, we are able to apply Corollary 3.4(a) from [11] to deduce that $f_{n}^{\prime}$ satisfies $\int_{0}^{t}\left\|f_{n}^{\prime}(s)\right\|^{p} \mathrm{~d} s<\infty$. Since $f_{n}^{\prime}$ is a measurable selection of $D_{H}(F)$, then $f_{n}^{\prime} \in S_{L^{p}}\left(D_{H}(F)\right)$. Therefore, $f_{n} \in \mathcal{I} \mathcal{S}(F)$ for every $n=1,2, \ldots$

\section{Set-Valued Young Integrals}

At the beginning of this section, we recall the notion of a Young integral in a singlevalued case introduced by Young in [30]. For details, see also [17]. Let $f:[0, T] \rightarrow$ $R^{d}$ and $g:[0, T] \rightarrow R^{d}$ be given functions. For the partition $\Pi_{m}: 0=t_{0}<t_{1}<$ $\cdots<t_{m}=T$ of the interval $[0, T]$, we consider the Riemann sum of $f$ with respect to $g$

$$
S\left(f, g, \Pi_{m}\right):=\sum_{i=1}^{m} f\left(t_{i-1}\right)\left(g\left(t_{i}\right)-g\left(t_{i-1}\right)\right) .
$$

Let $\left|\Pi_{m}\right|:=\max \left\{t_{i}-t_{i-1}: 1 \leq i \leq m-1\right\}$. Then, the following version of Proposition 2.4 in [18] holds.

Proposition 4 Let $f \in B \operatorname{Var}_{p}\left(R^{d}\right)$ and $g \in \mathcal{C}^{\alpha}\left(R^{1}\right)$ where $1 / p+\alpha>1$. Then, the limit

$$
\lim _{\left|\Pi_{m}\right| \rightarrow 0} S\left(f, g, \Pi_{m}\right)=: \int_{0}^{T} f \mathrm{~d} g
$$

exists and the inequality

$$
\left\|\int_{s}^{t} f \mathrm{~d} g-f(s)(g(t)-g(s))\right\| \leq C(\alpha, p)\left(\operatorname{Var}_{p}(f)\right)^{1 / p} M_{\alpha}(g)(t-s)^{\alpha}
$$

holds for every $0 \leq s<t \leq T$, where the constant $C(\alpha, p)$ depends only on $p$ and $\alpha$.

Corollary 1 Let $f_{1}, f_{2} \in B \operatorname{Var}_{p}\left(R^{d}\right)$ and $g \in \mathcal{C}^{\alpha}\left(R^{1}\right)$ where $1 / p+\alpha>1$. Then,

$$
\begin{aligned}
& \left\|\int_{0} f_{1} \mathrm{~d} g-\int_{0} f_{2} \mathrm{~d} g\right\|_{\alpha} \\
& \quad \leq\left(\left\|f_{1}-f_{2}\right\|_{\infty}+C(\alpha, p)\left(\operatorname{Var}_{p}\left(f_{1}-f_{2}\right)\right)^{1 / p}\right) M_{\alpha}(g)\left(1+T^{\alpha}\right) .
\end{aligned}
$$

In the case $f \in \mathcal{C}^{\beta}\left(R^{d}\right)$ and $\alpha, \beta \in(0,1]$ with $\alpha+\beta>1$, one can express the Young integral by fractional derivatives. Namely, let

$$
f_{0+}(t)=(f(t)-f(0+)) I_{(0, T)}(t) \text { and } f_{T-}(t)=(f(t)-f(T-)) I_{(0, T)}(t) .
$$


The right-sided and left-sided fractional derivatives of order $0<\rho<1$ for the function $f:[0, T] \rightarrow R^{1}$ are defined by

$$
D_{0+}^{\rho} f(t)=\frac{1}{\Gamma(1-\rho)}\left(\frac{f(t)}{t^{\rho}}+\rho \int_{0}^{t} \frac{f(t)-f(s)}{(t-s)^{\rho+1}} \mathrm{~d} s\right)
$$

and

$$
D_{T-}^{\rho} f(t)=\frac{(-1)^{\rho}}{\Gamma(1-\rho)}\left(\frac{f(t)}{(T-t)^{\rho}}+\rho \int_{t}^{T} \frac{f(t)-f(s)}{(s-t)^{\rho+1}} \mathrm{~d} s\right) .
$$

Then, the following result holds, see, e.g., [28].

Proposition 5 Suppose that $g:[0, T] \rightarrow R^{1}, g \in \mathcal{C}^{\alpha}\left(R^{1}\right)$ and $f \in \mathcal{C}^{\beta}\left(R^{d}\right)$. Then, the integral $\int_{0}^{T} f \mathrm{~d} g$ exists in the sense of Riemann and

$$
\int_{0}^{T} f \mathrm{~d} g=(-1)^{\rho} \int_{0}^{T} D_{0+}^{\rho} f_{0+}(t) D_{T-}^{1-\rho} g_{T-}(t) \mathrm{d} t+f(0)(g(T)-g(0))
$$

for every $\rho \in(1-\alpha, \beta)$. Moreover, the following version of inequality (8)

$$
\left\|\int_{t_{1}}^{t_{2}} f \mathrm{~d} g-f\left(t_{1}\right)\left(g\left(t_{2}\right)-g\left(t_{1}\right)\right)\right\| \leq C(\alpha, \beta) M_{\alpha}(g) M_{\beta}(f)\left(t_{2}-t_{1}\right)^{\alpha+\beta}
$$

holds for every $0 \leq t_{1}<t_{2} \leq T$, where $C(\alpha, \beta)$ depends only on $\alpha$ and $\beta$.

Let us consider again a set $\mathcal{I S}(F)$ given in (3)

$$
\mathcal{I S}(F)=\left\{f \in B V_{p}\left(R^{d}\right): f \in S_{V_{p}}(F) \text { and } f^{\prime} \in S_{L^{p}}\left(D_{H}(F)\right)\right\} .
$$

Definition 5 We define a set-valued Young integral of Hukuhara differentiable $F \in$ $B V_{p}\left(\operatorname{Conv} R^{n}\right)$ with respect to a function $g \in \mathcal{C}^{\alpha}\left(R^{1}\right), 1 / p+\alpha>1$, by the formula

$$
(\mathcal{I S}) \int_{0}^{t} F \mathrm{~d} g:=c l_{R^{d}}\left\{\int_{0}^{t} f \mathrm{~d} g: f \in \mathcal{I} \mathcal{S}(F)\right\},
$$

for every $1 / p+\alpha>1$ and $g \in C^{\alpha}\left(R^{1}\right)$.

We have

$$
\left\|(\mathcal{I S}) \int_{s}^{t} F \mathrm{~d} g\right\| \leq M_{\alpha}(g)\|F\|_{\beta}\left(1+C(\alpha, \beta) T^{\beta}\right)(t-s)^{\alpha}
$$

for $0 \leq s \leq t \leq T$. Since $F$ and $D_{H}(F)$ take on convex values, then the sets $S V_{p}(F)$ and $S_{L^{p}}\left(D_{H}(F)\right)$ are convex and therefore, $\mathcal{I} \mathcal{S}(F)$ and $(\mathcal{I S}) \int_{0}^{t} F \mathrm{~d} g$ for every $t \in[0, T]$ are convex subsets of $B V_{p}\left(R^{d}\right)$ and $R^{d}$, respectively.

The following lemma was proved in [27]. 
Lemma 1 Let $g \in \mathcal{C}^{\alpha}\left(R^{1}\right)$. Then, for every $\rho \in(1-\alpha, \beta)$, there exists a positive constant $C(\rho)$ such that for every $f_{1}, f_{2} \in \mathcal{C}^{\beta}\left(R^{d}\right), t \in[0, T]$ and $\theta \in(0,1]$ the inequality

$$
\begin{aligned}
\left\|\int_{0}^{t} f_{1} \mathrm{~d} g-\int_{0}^{t} f_{2} \mathrm{~d} g\right\| \leq & C(\rho)\left[\left\|f_{1}-f_{2}\right\|_{\infty}+\left(M_{\beta}\left(f_{1}\right)+M_{\beta}\left(f_{2}\right)\right) \theta^{\beta}\right] \theta^{-\rho} \\
& +\left\|f_{1}(0)-f_{2}(0)\right\||g(T)-g(0)| .
\end{aligned}
$$

holds.

Using this lemma, we are able to prove the following result.

Theorem 6 For every $\rho \in(1-\alpha, \beta)$, there exists a positive constant $C(\rho)$ such that for every $\theta \in(0,1], t \in[0, T]$ and for every Hukuhara differentiable set-valued functions $F_{1}, F_{2}$ with bounded Hukuhara derivatives, the inequality

$$
\begin{aligned}
& H_{R^{d}}\left((\mathcal{I S}) \int_{0}^{t} F_{1} \mathrm{~d} g,(\mathcal{I} \mathcal{S}) \int_{0}^{t} F_{2} \mathrm{~d} g\right) \\
& \leq C(\rho)\left(\int_{0}^{T} H_{R^{d}}\left(D_{H}\left(F_{1}\right)(s), D_{H}\left(F_{2}\right)(s)\right) \mathrm{d} s\right. \\
& \left.\quad+\left(T+T^{1-\beta}\right)\left(\sup _{t \in[0, T]}\left\|D_{H}\left(F_{1}\right)(t)\right\|+\sup _{t \in[0, T]}\left\|D_{H}\left(F_{2}\right)(t)\right\|\right) \theta^{\beta}\right) \theta^{-\rho} \\
& \quad+M_{\alpha}(g) T^{\alpha} \int_{0}^{T} H_{R^{d}}\left(D_{H}\left(F_{1}\right)(s), D_{H}\left(F_{2}\right)(s)\right) \mathrm{d} s .
\end{aligned}
$$

holds.

Proof Let $F:[0, T] \rightarrow \operatorname{Conv}\left(R^{d}\right)$ be Hukuhara differentiable. If the setvalued function $D_{H}(F)(\cdot)$ is bounded, i.e., $\sup _{t \in[0, T]}\left\|D_{H}(F)(t)\right\|<\infty$, then $\|F\|_{\infty} \leq T \sup _{t \in[0, T]}\left\|D_{H}(F)(t)\right\|$ and $V_{p}(F) \leq T \sup _{t \in[0, T]}\left\|D_{H}(F)(t)\right\|^{p}$ as well as $M_{\beta}(F) \leq T^{1-\beta} \sup _{t \in[0, T]}\left\|D_{H}(F)(t)\right\|$ for any $p \geq 1$ and $\beta \in(0,1)$. Thus $F \in B V_{p}\left(\operatorname{Conv}\left(R^{d}\right)\right)$ and $S_{V_{p}}(F) \neq \emptyset$.

We obtain by Lemma 1 , for any $f_{1} \in \mathcal{I S}\left(F_{1}\right), f_{2} \in, \mathcal{I S}\left(F_{2}\right), \rho \in(1-\alpha, \beta)$, $t \in[0, T]$ and $\theta \in(0,1]$

$$
\begin{aligned}
& \left\|\int_{0}^{t} f_{1} \mathrm{~d} g-\int_{0}^{t} f_{2} \mathrm{~d} g\right\| \\
& \quad \leq C(\rho)\left[\left\|f_{1}-f_{2}\right\|_{\infty}+\left(M_{\beta}\left(f_{1}\right)+M_{\beta}\left(f_{2}\right)\right) \theta^{\beta}\right] \theta^{-\rho}+\left\|f_{1}-f_{2}\right\|_{\infty} M_{\alpha}(g) T^{\alpha} .
\end{aligned}
$$


Thus,

$$
\begin{aligned}
& \operatorname{dist}_{R^{d}}\left(\int_{0}^{t} f_{1} \mathrm{~d} g,(\mathcal{I} \mathcal{S}) \int_{0}^{t} F_{2} \mathrm{~d} g\right) \\
& \leq C(\rho)\left[\operatorname{dist}_{\infty}\left(f_{1}, \mathcal{I} \mathcal{S}\left(F_{2}\right)\right)+\left(\sup _{f_{1} \in \mathcal{I} \mathcal{S}\left(F_{1}\right)} M_{\beta}\left(f_{1}\right)+\sup _{f_{2} \in \mathcal{I} \mathcal{S}\left(F_{2}\right)} M_{\beta}\left(f_{2}\right)\right) \theta^{\beta}\right] \theta^{-\rho} \\
& \quad+\operatorname{dist}_{\infty}\left(f_{1}, \mathcal{I} \mathcal{S}\left(F_{2}\right)\right) M_{\alpha}(g) T^{\alpha} .
\end{aligned}
$$

Hence,

$$
\begin{aligned}
& \bar{H}_{R^{d}}\left((\mathcal{I S}) \int_{0}^{t} F_{1} \mathrm{~d} g,(\mathcal{I S}) \int_{0}^{t} F_{2} \mathrm{~d} g\right) \\
& \leq C(\rho)\left[H_{\infty}\left(\mathcal{I} \mathcal{S}\left(F_{1}\right), \mathcal{I} \mathcal{S}\left(F_{2}\right)\right)+\left(\sup _{f_{1} \in \mathcal{I} \mathcal{S}\left(F_{1}\right)} M_{\beta}\left(f_{1}\right)+\sup _{f_{2} \in \mathcal{I} \mathcal{S}\left(F_{2}\right)} M_{\beta}\left(f_{2}\right)\right) \theta^{\beta}\right] \theta^{-\rho} \\
& \quad+M_{\alpha}(g) T^{\alpha} H_{\infty}\left(\mathcal{I S}\left(F_{1}\right), \mathcal{I S}\left(F_{2}\right)\right) .
\end{aligned}
$$

The same estimation holds for $\bar{H}_{R^{d}}\left((\mathcal{I S}) \int_{0}^{t} F_{1} \mathrm{~d} g,(\mathcal{I S}) \int_{0}^{t} F_{2} \mathrm{~d} g\right)$.

Therefore,

$$
\begin{aligned}
& H_{R^{d}}\left((\mathcal{I} \mathcal{S}) \int_{0}^{t} F_{1} \mathrm{~d} g,(\mathcal{I} \mathcal{S}) \int_{0}^{t} F_{2} \mathrm{~d} g\right) \\
& \leq C(\rho)\left[H_{\infty}\left(\mathcal{I} \mathcal{S}\left(F_{1}\right), \mathcal{I S}\left(F_{2}\right)\right)+\left(\sup _{f_{1} \in \mathcal{I} \mathcal{S}\left(F_{1}\right)} M_{\beta}\left(f_{1}\right)+\sup _{f_{2} \in \mathcal{I} \mathcal{S}\left(F_{2}\right)} M_{\beta}\left(f_{2}\right)\right) \theta^{\beta}\right] \theta^{-\rho} \\
& \quad+M_{\alpha}(g) T^{\alpha} H_{\infty}\left(\mathcal{I} \mathcal{S}\left(F_{1}\right), \mathcal{I S}\left(F_{2}\right)\right) .
\end{aligned}
$$

Suppose that $f \in \mathcal{I S}(F)$. Then, it is expressed as the integral, i.e., $f(\cdot)=\int_{0}^{\cdot} \phi(s) \mathrm{d} s$ for some $\phi \in S_{L^{p}}\left(D_{H}(F)\right)$ and we have

$$
M_{\beta}(f)=\sup _{0 \leq s<t \leq T} \frac{\|f(t)-f(s)\|}{(t-s)^{\beta}}=\sup _{0 \leq s<t \leq T} \frac{\left\|\int_{s}^{t} \phi(\tau) \mathrm{d} \tau\right\|}{(t-s)^{\beta}} .
$$

From the other side, we get by formula (2), the equalities

$$
\begin{aligned}
M_{\beta}(F) & =\sup _{0 \leq s<t \leq T} \frac{H_{R^{d}}(F(t), F(s))}{(t-s)^{\beta}} \\
& =\sup _{0 \leq s<t \leq T} \frac{H_{R^{d}}\left(\int_{s}^{t} D_{H} F(\tau) \mathrm{d} \tau+\int_{0}^{s} D_{H} F(\tau) \mathrm{d} \tau, 0+\int_{0}^{s} D_{H} F(\tau) \mathrm{d} \tau\right)}{(t-s)^{\beta}} \\
& =\sup _{0 \leq s<t \leq T} \frac{H_{R^{d}}\left(\int_{s}^{t} D_{H} F(\tau) \mathrm{d} \tau, 0\right)}{(t-s)^{\beta}}=\sup _{0 \leq s<t \leq T} \frac{\left\|\int_{s}^{t} D_{H} F(\tau) \mathrm{d} \tau\right\|}{(t-s)^{\beta}}
\end{aligned}
$$


Therefore,

$$
M_{\beta}(f) \leq M_{\beta}(F)
$$

and taking in the mind the beginning of the proof, we get

$$
\sup _{f \in \mathcal{I} \mathcal{S}(F)}\|f\|_{\beta} \leq\|F\|_{\beta} \leq\left(T+T^{1-\beta}\right) \sup _{t \in[0, T]}\left\|D_{H}(F)(t)\right\|<\infty .
$$

Let $\phi_{1} \in S_{L^{p}}\left(D_{H}\left(F_{1}\right)\right)$. Then, by Theorem 2.2 from [20], we have

$$
\begin{aligned}
& \inf _{\phi_{2} \in S_{L} p\left(D_{H}\left(F_{2}\right)\right)} \sup _{t \in[0, T]}\left\|\int_{0}^{t} \phi_{1}(s) \mathrm{d} s-\int_{0}^{t} \phi_{2}(s) \mathrm{d} s\right\| \\
& \leq \inf _{\phi_{2} \in S_{L} p\left(D_{H}\left(F_{2}\right)\right)} \int_{0}^{T}\left\|\phi_{1}(s)-\phi_{2}(s)\right\| \mathrm{d} s=\int_{0}^{T} \operatorname{dist}_{R^{d}}\left(\phi_{1}(s), D_{H}\left(F_{2}\right)(s)\right) \mathrm{d} s \\
& \leq \int_{0}^{T} H_{R^{d}}\left(D_{H}\left(F_{1}\right)(s), D_{H}\left(F_{2}\right)(s)\right) \mathrm{d} s
\end{aligned}
$$

Thus,

$$
\bar{H}_{\infty}\left(\mathcal{I S}\left(F_{1}\right), \mathcal{I} \mathcal{S}\left(F_{2}\right)\right) \leq \int_{0}^{T} H_{R^{d}}\left(D_{H}\left(F_{1}\right)(s), D_{H}\left(F_{2}\right)(s)\right) \mathrm{d} s
$$

In a similar way, we get

$$
\bar{H}_{\infty}\left(\mathcal{I} \mathcal{S}\left(F_{2}\right), \mathcal{I} \mathcal{S}\left(F_{1}\right)\right) \leq \int_{0}^{T} H_{R^{d}}\left(D_{H}\left(F_{1}\right)(s), D_{H}\left(F_{2}\right)(s)\right) \mathrm{d} s
$$

and finally

$$
H_{\infty}\left(\mathcal{I} \mathcal{S}\left(F_{1}\right), \mathcal{I} \mathcal{S}\left(F_{2}\right)\right) \leq \int_{0}^{T} H_{R^{d}}\left(D_{H}\left(F_{1}\right)(s), D_{H}\left(F_{2}\right)(s)\right) \mathrm{d} s .
$$

Hence, by formula (10) together with (11), we obtain inequality (9).

Corollary 2 Let $F_{n}, F$ be Hukuhara differentiable set-valued functions with bounded Hukuhara derivatives satisfying

$$
\sup _{t \in[0, T]} H_{R^{d}}\left(D_{H}\left(F_{n}\right)(t), D_{H}(F)(t)\right) \rightarrow 0 \text { as } n \rightarrow \infty \text {. }
$$

Then,

$$
\sup _{t \in[0, T]} H_{R^{d}}\left((\mathcal{I S}) \int_{0}^{t} F_{n} \mathrm{~d} g,(\mathcal{I S}) \int_{0}^{t} F \mathrm{~d} g\right) \rightarrow 0 \text { as } n \rightarrow \infty
$$


Proof Let us note that for every $n \geq 1$ we have

$$
\begin{aligned}
& \left|\sup _{t \in[0, T]}\left\|D_{H}\left(F_{n}\right)(t)\right\|-\sup _{t \in[0, T]}\left\|D_{H}(F)(t)\right\|\right| \\
& \quad \leq \sup _{t \in[0, T]} H_{R^{d}}\left(D_{H}\left(F_{n}\right)(t), D_{H}(F)(t)\right) .
\end{aligned}
$$

Thus,

$$
\sup _{t \in[0, T]}\left\|D_{H}\left(F_{n}\right)(t)\right\| \rightarrow \sup _{t \in[0, T]}\left\|D_{H}(F)(t)\right\| \text { as } n \rightarrow \infty
$$

Therefore, the sequence $\left(\sup _{t \in[0, T]}\left\|D_{H}\left(F_{n}\right)(t)\right\|\right)_{n \geq 1}$ is bounded. Hence, we get by Theorem 6

$$
\begin{aligned}
& \underset{n}{\limsup }\left(\sup _{t \in[0, T]} H_{R^{d}}\left((\mathcal{I S}) \int_{0}^{t} F_{n} \mathrm{~d} g,(\mathcal{I S}) \int_{0}^{t} F \mathrm{~d}\right)\right) \\
& \leq C(\rho)\left(T+T^{1-\beta}\right)\left(\sup _{n} \sup _{t \in[0, T]}\left\|D_{H}\left(F_{n}\right)(t)\right\|+\sup _{t \in[0, T]}\left\|D_{H}(F)(t)\right\|\right) \theta^{\beta-\rho} .
\end{aligned}
$$

Since $\beta>\rho$ and $\theta \in(0,1]$ is arbitrarily taken, we obtain formula (12).

It was proved in [20] that for measurable and $p$-integrably bounded set-valued functions $F_{1}, F_{2}:[0, T] \rightarrow \operatorname{Comp}\left(R^{d}\right)$, the equality

$$
\left.S_{L^{p}}\left(c l_{R^{d}}\left\{F_{1}+F_{2}\right)(\cdot)\right\}\right)=\operatorname{cl}_{L^{p}}\left\{S_{L^{p}}\left(F_{1}\right)+S_{L^{p}}\left(F_{2}\right)\right\}
$$

holds. Therefore, a set-valued Aumann integral satisfies

$$
\int_{0}^{t}\left(F_{1}+F_{2}\right) \mathrm{d} t=\int_{0}^{t} F_{1} \mathrm{~d} t+\int_{0}^{t} F_{2} \mathrm{~d} t
$$

We will show that a set-valued Young integral is additive also.

Theorem 7 Let $F, F_{1}, F_{2} \in B V_{p}\left(\operatorname{Conv}\left(R^{d}\right)\right)$ be Hukuhara differentiable with $p$ integrably bounded Hukuhara derivatives, $1<p<\infty$. Let $g \in C^{\alpha}\left(R^{1}\right)$, where $1 / p+\alpha>1$. Then,

$$
(\mathcal{I S}) \int_{0}^{t}\left(F_{1}+F_{2}\right) \mathrm{d} g=(\mathcal{I S}) \int_{0}^{t} F_{1} \mathrm{~d} g+(\mathcal{I S}) \int_{0}^{t} F_{2} \mathrm{~d} g .
$$

Moreover, if the set $F(0)$ is bounded in $R^{d}$, then $(\mathcal{I S}) \int_{0}^{\cdot} F \mathrm{~d} g$ and $(\mathcal{I S}) \int_{S}^{t} F \mathrm{~d} g$ are bounded sets in $C^{\alpha}\left(R^{d}\right)$ and in $R^{d}$, respectively. 
Proof We show that $\mathcal{I S}\left(F_{1}+F_{2}\right)=\mathcal{I S}\left(F_{1}\right)+\mathcal{I S}\left(F_{2}\right)$. Let us take an arbitrary $f \in \mathcal{I} \mathcal{S}\left(F_{1}+F_{2}\right)$. Then, $f \in S V_{p}\left(F_{1}+F_{2}\right), f^{\prime} \in S_{L^{p}}\left(D_{H}\left(F_{1}+F_{2}\right)\right)$ and $f(\cdot)=\int_{0}^{\cdot} f^{\prime}(s) \mathrm{d} s$. Since $D_{H}\left(F_{i}\right)(t)$ takes on compact and convex values in $R^{d}$, then $f^{\prime} \in S_{L^{p}}\left(D_{H}\left(F_{1}+F_{2}\right)\right)=c l_{L^{p}}\left\{S_{L^{p}}\left(D_{H}\left(F_{1}\right)\right)+S_{L^{p}}\left(D_{H}\left(F_{2}\right)\right)\right\}$ by equality (13). Therefore, there exist sequences $\left(\phi_{n}^{1}\right) \subset S_{L^{p}}\left(D_{H}\left(F_{1}\right)\right)$ and $\left(\phi_{n}^{2}\right) \subset$ $S_{L^{p}}\left(D_{H}\left(F_{2}\right)\right)$ such that $\phi_{n}^{1}+\phi_{n}^{2} \rightarrow f^{\prime}$ with respect to the $L^{p}$-norm convergence. But $S_{L^{p}}\left(D_{H}\left(F_{1}\right)\right)$ is a closed, convex and bounded subset of $L^{p}$ by [20], and therefore, weakly compact. Then, there exists a subsequence $\left(\phi_{n_{k}}^{1}\right)$ weakly convergent to some $\phi^{1} \in S_{L^{p}}\left(D_{H}\left(F_{1}\right)\right)$. Similarly, passing to the subsequence if needed, $\left(\phi_{n_{k}}^{2}\right)$ tends weakly to some $\phi^{2} \in S_{L^{p}}\left(D_{H}\left(F_{2}\right)\right)$. Therefore, $f^{\prime}=\phi^{1}+\phi^{2} \in$ $S_{L^{p}}\left(D_{H}\left(F_{1}\right)\right)+S_{L^{p}}\left(D_{H}\left(F_{2}\right)\right)$. But $f(\cdot)=\int_{0}^{\cdot} f^{\prime}(s) \mathrm{d} s=\int_{0}^{\cdot} \phi^{1}(s) \mathrm{d} s+\int_{0}^{\cdot} \phi^{2}(s) \mathrm{d} s$. Since $\int_{0}^{t} \phi^{1}(s) \mathrm{d} s \in \int_{0}^{t} D_{H}\left(F_{1}\right)(s) \mathrm{d} s=F_{1}(t)$, then $\int_{0}^{*} \phi^{1}(s) \mathrm{d} s \in \mathcal{I} \mathcal{S}\left(F_{1}\right)$. In the same way, $\int_{0} \phi^{2}(s) \mathrm{d} s \in \mathcal{I S}\left(F_{2}\right)$, and therefore, $\mathcal{I S}\left(F_{1}+F_{2}\right) \subset \mathcal{I} \mathcal{S}\left(F_{1}\right)+\mathcal{I S}\left(F_{2}\right)$. For the proof of a reverse inclusion, it is enough to note that taking $f_{1} \in \mathcal{I S}\left(F_{1}\right)$ and $f_{2} \in \mathcal{I S}\left(F_{2}\right)$, their sum belongs to $S V_{p}\left(F_{1}+F_{2}\right)$ and the sum of their derivatives belongs to $S_{L^{p}}\left(D_{H}\left(F_{1}+F_{2}\right)\right)$. Hence, $f_{1}+f_{2} \in \mathcal{I} \mathcal{S}\left(F_{1}+F_{2}\right)$.

Now let us remark that the operator $J: B V_{p}\left(R^{d}\right) \rightarrow R^{d}$ defined by the formula $J(f)=\int_{0}^{t} f \mathrm{~d} g$ is linear. From this, we get

$$
\begin{aligned}
& (\mathcal{I S}) \int_{0}^{t}\left(F_{1}+F_{2}\right) \mathrm{d} g=J\left(\mathcal{I S}\left(F_{1}+F_{2}\right)\right)=J\left(\mathcal{I S}\left(F_{1}\right)+\mathcal{I S}\left(F_{2}\right)\right) \\
& =J\left(\mathcal{I S}\left(F_{1}\right)\right)+J\left(\mathcal{I S}\left(F_{2}\right)\right)=(\mathcal{I S}) \int_{0}^{t}\left(F_{1}\right) \mathrm{d} g+(\mathcal{I S}) \int_{0}^{t}\left(F_{2}\right) \mathrm{d} g .
\end{aligned}
$$

Hence, the first statement follows.

We show that the set $\mathcal{I} \mathcal{S}(F)$ is bounded in the space $\left(B V_{p}\left(R^{d}\right),\|\cdot\|_{V_{p}}\right)$. Let $f \in \mathcal{I} \mathcal{S}(F)$ be arbitrarily taken. By assumption, the set $S_{L^{p}}\left(D_{H}(F)\right)$ is bounded in $L^{p}$ norm by some constant $M$. Since $f^{\prime} \in S_{L^{p}}\left(D_{H}(F)\right)$, then $\left(V_{p}(f)\right)^{1 / p}=$ $\left(\int_{0}^{T}\left\|f^{\prime}(s)\right\|^{p} \mathrm{~d} s\right)^{1 / p} \leq M$.

Moreover, $\|f(t)-f(0)\| \leq T^{1-1 / p}\left(V_{p}(f)\right)^{1 / p}$ for every $t \in[0, T]$ by Proposition 1(b). This implies

$$
\|f\|_{V_{p}}=\|f\|_{\infty}+\left(V_{p}(f)\right)^{1 / p} \leq \sup _{h \in \mathcal{I} \mathcal{S}(F)}\|h(0)\|+T^{1-1 / p} M+M
$$

Then, for every $f \in \mathcal{I S}(F)$, we get by Corollary 1

$$
\begin{aligned}
& \left\|\int_{0} f \mathrm{~d} g\right\|_{\alpha} \leq\left(\|f\|_{\infty}+C(\alpha, p)\left(\operatorname{Var}_{p}(f)\right)^{1 / p}\right) M_{\alpha}(g)\left(1+T^{\alpha}\right) \\
& \quad \leq \sup _{h \in \mathcal{I} \mathcal{S}(F)}\|h(0)\|+\left(T^{1-1 / p}\right) M+C(\alpha, p) M T^{1-1 / p} M_{\alpha}(g)\left(1+T^{\alpha}\right) \\
& =\sup _{h \in \mathcal{I} \mathcal{S}(F)}\|h(0)\|+\left(T^{1-1 / p}\right) M\left(1+C(\alpha, p) M_{\alpha}(g)\left(1+T^{\alpha}\right)\right) .
\end{aligned}
$$


Using Corollary 1 once again, we obtain in a similar way

$$
\begin{aligned}
& \left\|\int_{s}^{t} f \mathrm{~d} g\right\| \leq\left(\|f\|_{\infty}+C(\alpha, p)\left(\operatorname{Var}_{p}(f)\right)^{1 / p}\right) M_{\alpha}(g)(t-s)^{\alpha} \\
& \quad \leq \sup _{h \in \mathcal{I} \mathcal{S}(F)}\|h(0)\|+\left(T^{1-1 / p}\right) M\left(1+C(\alpha, p) M_{\alpha}(g) T^{\alpha}\right) .
\end{aligned}
$$

Thus, we obtain the appropriate boundedness of both integrals.

\section{Compliance with Ethical Standards}

Conflict of interest The authors declare that they have no conflict of interest.

Open Access This article is licensed under a Creative Commons Attribution 4.0 International License, which permits use, sharing, adaptation, distribution and reproduction in any medium or format, as long as you give appropriate credit to the original author(s) and the source, provide a link to the Creative Commons licence, and indicate if changes were made. The images or other third party material in this article are included in the article's Creative Commons licence, unless indicated otherwise in a credit line to the material. If material is not included in the article's Creative Commons licence and your intended use is not permitted by statutory regulation or exceeds the permitted use, you will need to obtain permission directly from the copyright holder. To view a copy of this licence, visit http://creativecommons.org/licenses/by/4.0/.

\section{References}

1. Ahmed, N.U.: Semigroup Theory with Applications to Systems and Control. Pitman Research Notes in Mathematical Series. Longman, Harlow (1991)

2. Ahmed, N.U.: Optimal Relaxed Controls for Nonlinear Infinite Dimensional Stochastic Differential Inclusions. Lecture Notes in Pure and Applied Mathematics, vol. 180, pp. 1-19. Marcel Dekker, New York (1994)

3. Aubin, J.P.: Viability Theory. Birkhäuser, Boston (2009)

4. Aubin, J.P., Cellina, A.: Differential Inclusions. Springer, Berlin (1984)

5. Aubin, J.P., Frankowska, H.: Set-Valued Analysis. Birkhäuser, Boston (1990)

6. Aumann, R.J.: Integrals of set-valued functions. J. Math. Anal. Appl. 12, 1-12 (1965)

7. Bailleul, I., Brault, A., Coutin, L.: Young and rough differential inclusions. arXiv: 1812.06727v2 [math.CA] (5 Jun 2019)

8. Baier, R., Farkhi, E.: Regularity and integration of set-valued maps represented by generalized Steiner points. Set Valued Anal. 15, 185-207 (2007)

9. Castaing, C.: Sur l'existence des sections séparément mesurables et séparément continues d'une multiapplication, Séminaire d'Analyse Convexe, Univ. des Sci. et Techniques du Languedoc Montpellier, Expose No. 14 (1975)

10. Chistyakov, V.V., Galkin, O.E.: On maps of bounded $p$-variation with $p>1$. Positivity 2, 19-45 (1998)

11. Chistyakov, V.: Selections of bounded variation. J. Appl. Anal. 10(1), 1-82 (2004)

12. Coutin, L., Qian, Z.: Stochastic analysis, rough paths analysis and fractional Brownian motions. Probab. Theory Relat. Fields 122, 108-140 (2002)

13. Dentcheva, D.: Differentiable selections and Castaing representations of multifunctions. J. Math. Anal. Appl. 223, 371-396 (1998)

14. Diamond, P., Kloeden, P.: Metric Spaces of Fuzzy Sets: Theory and Applications. World Scientific, Singapore (1994)

15. Djebali, S., Górniewicz, L., Ouahab, A.: Solutions Sets for Differential Equations and Inclusions. Series in Nonlinear Analysis and Applications. De Gruyter GmbH, Berlin (2013) 
16. Fei, W.Y., Xia, D.F.: On solutions to stochastic set differential equations of Itô type under the nonLipschitzian condition. Dyn. Syst. Appl. 22(1), 137-156 (2013)

17. Friz, P.K., Victoir, N.B.: Multidimensional Stochastic Processes as Rough Paths: Theory and Applications. Cambridge Studies in Advanced Mathematics. Cambridge University Press, Cambridge (2010)

18. Friz, P.K., Zhang, H.: Differential equations driven by rough paths with jumps. J. Differ. Equ. 264, 6226-6301 (2018)

19. Fryszkowski, A.: Fixed Point Theory for Decomposable Sets. Kluwer, Dordrecht (2004)

20. Hiai, F., Umegaki, H.: Integrals, conditional expectations and martingales for multivalued functions. J. Multivar. Anal. 7(1), 149-182 (1977)

21. Kisielewicz, M.: Differential Inclusions and Optimal Control. Kluwer, Dordrecht (1991)

22. Kisielewicz, M.: Stochastic Differential Inclusions and Applications. Springer, New York (2013)

23. Lakshmikantham, V., Gnana Bhaskar, T., Vasundhara Devi, J.: Theory of Set Differential Equations in a Metric Space. Cambridge Scientific Publishers, Cambridge (2006)

24. Lakshmikantham, V., Tolstonogov, A.A.: Existence and interrelation between set and fuzzy differential equations. Nonlinear Anal. 55, 255-268 (2003)

25. Lejay, A.: Controlled differential equations as Young integrals: a simple approach. J. Differ. Equ. 249, 1777-1798 (2010)

26. Lyons, T.: Differential equations driven by rough signals. Rev. Mat. Iberoam. 14, 215-310 (1998)

27. Michta, M., Motyl, J.: Selections properties and set-valued Young integrals of set-valued functions. Results Math. 75, 164 (2020). https://doi.org/10.1007/s00025-020-01284-3

28. Samko, S.G., Kilbas, A.A., Marichev, O.I.: Fractional Integrals and Derivatives, Theory and Applications. Gordon and Breach Science Publishers, Yverdon (1993)

29. Tolstonogov, A.A.: Differential Inclusions in a Banach Space. Kluwer, Dordrecht (2000)

30. Young, L.S.: An inequality of the Hölder type connected with Stieltjes integration. Acta Math. 67, 251-282 (1936)

Publisher's Note Springer Nature remains neutral with regard to jurisdictional claims in published maps and institutional affiliations. 